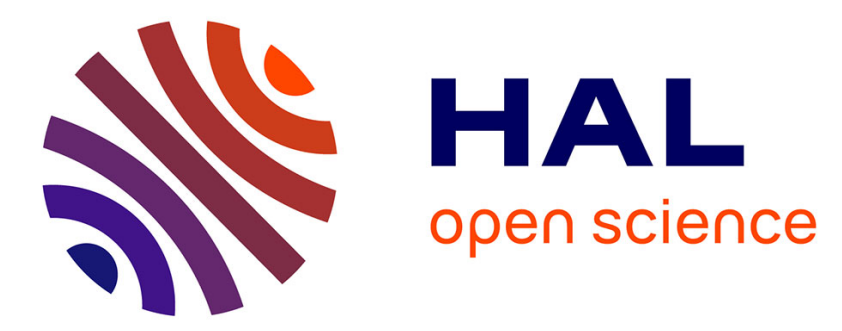

\title{
Comportement d'une couche labourée sous des actions de compactage en fonction de son état hydrique et structural
}

\author{
François Papy, Christophe Maitre
}

\section{- To cite this version:}

François Papy, Christophe Maitre. Comportement d'une couche labourée sous des actions de compactage en fonction de son état hydrique et structural. Agronomie, 1987, 7 (2), pp.111-121. hal00884975

\section{HAL Id: hal-00884975 \\ https://hal.science/hal-00884975}

Submitted on 1 Jan 1987

HAL is a multi-disciplinary open access archive for the deposit and dissemination of scientific research documents, whether they are published or not. The documents may come from teaching and research institutions in France or abroad, or from public or private research centers.
L'archive ouverte pluridisciplinaire HAL, est destinée au dépôt et à la diffusion de documents scientifiques de niveau recherche, publiés ou non, émanant des établissements d'enseignement et de recherche français ou étrangers, des laboratoires publics ou privés. 


\title{
Comportement d'une couche labourée sous des actions de compactage en fonction de son état hydrique et structural
}

\author{
François PAPY \\ avec la collaboration technique de Christophe MAITRE
}

I.N.R.A. - Unité de Recherche sur les Systèmes Agraires et le Développement, Centre de Recherches de ParisGrignon, $F 78850$ Thiverval-Grignon

Mots clés additionnels : Profil cultural, comportement mécanique du sol, porosité structurale, porosité texturale, morphométrie.

The behaviour of the ploughed layer towards compaction according to its water content and structure

The compaction of the ploughed layer by agricultural machinery was investigated according to the water content and structure of the layer. The structural state of the profile was characterized by measures of bulk density as well as by quantifiable morphological description. By combination of these two indices, together with the results of an analysis of shrinkage-swelling curves made on small compact aggregates (of so-called $\Delta$ type), an analysis of the porosity system could be made.

With increasing moisture content and compaction energy, the following phenomena were observed : increasing compactness of the ploughed layer at right angles to the contact surface, first for the assemblage of structural elements, then for their internal state. This last manifestation was accompanied by an increase of matter flow. The effect of the initial structural state was expressed through its specific behaviour, in relation to moisture content as well as by the arrangement of the morphological units. This investigation demonstrates the power of morphometric examination.

Additional key words : Cultural profile, mechanical behaviour, structural porosity, textural porosity, morphometry. 


\section{INTRODUCTION}

Entre une récolte et l'installation de la culture suivante l'état physique du sol subit une évolution lente et progressive sous l'action du climat, et généralement brutale sous l'action des opérations techniques de travail du sol (MANICHON \& SEBILlOTTE, 1975).

Ayant apporté une contribution à l'étude de l'effet de l'état structural sur le comportement hydrique du sol sous l'effet de séquences de pluie et de dessication (PAPY, 1984, 1986a), nous allons présenter ici des résultats expérimentaux relatifs à l'action des façons culturales. Ils portent sur l'effet de compactage dus aux roues d'engins agricoles, étudié en fonction de l'état hydrique et structural du profil cultural au moment de l'intervention.

De nombreux travaux ont été réalisés sur ce sujet, dont une revue bibliographique a été dressée récemment (SOANE et al., 1981). Ils s'appliquent à des situations où la structure est plus homogène que celle observée généralement dans les conditions de la pratique. En effet, la couche travaillée du sol se caractérise souvent par une variabilité interne de l'état structural et des discontinuités par rapport à des directions verticales et horizontales (MANICHON, 1986) que nous avons décidé de prendre en compte.

Dans la plupart des travaux de la littérature agronomique, l'effet de la structure de la couche travaillée n'est pas étudié comme facteur de variation des actions de compactage. En réalisant sur deux types structuraux de couche labourée des compactages à différentes dates après des séquences de pluie ayant ramené le sol à la capacité au champ, nous allons analyser le rôle de l'état physique initial sur le résultat de ces actions. Ainsi que le propose MANiCHON (1982b), nous définissons l'état physique par ses deux composantes : l'état hydrique et l'état structural.

\section{MATÉRIELS ET MÉTHODES}

\section{A. Caractéristiques du sol et des types structuraux de labour}

L'expérience a été réalisée, au cours du printemps 1983, dans une parcelle du domaine expérimental INRA-INA/PG de GRIGNON (Yvelines), sur un sol d'alluvions limono-argileux perméable dont les caractéristiques sont données au tableau 1. Deux situations ont été comparées :

- la première où les bandes de terre découpées par la charrue ont été fortement fragmentées ; les éléments structuraux ont une taille maximale de l'ordre du centimètre et sont facilement discernables les unes des autres : nous l'appelons couche de type $\mathrm{O}$;

- la seconde où la terre, compactée à l'état humide avant labour, a donné, sous l'action du corps de charrue, des bandes peu fragmentées formant des blocs compacts : elle est dénommée couche de type B.

Ces situations représentent, selon MANICHON (1986), les deux modalités types auxquelles on peut rattacher les couches de sol après labour avant
TABLEAU

Caractéristiques granulométriques du sol étudié. Mean characteristics of the soil used.

\begin{tabular}{|c|c|c|c|c|c|c|c|}
\hline Horizon & $\begin{array}{c}\text { A } \\
<2 \mu\end{array}$ & $\begin{array}{c}1 \\
2-20 \mu\end{array}$ & $\begin{array}{c}\mathrm{L} \\
20-50 \mu\end{array}$ & $\stackrel{s}{50-200 \mu}$ & $\begin{array}{c}S \\
200- \\
2000 \mu\end{array}$ & $\begin{array}{l}\text { Mat. } \\
\text { Org. } \\
(\%)\end{array}$ & $\begin{array}{c}\text { Carbo- } \\
\text { nates } \\
(0 \% 0)\end{array}$ \\
\hline Couche labourée & 27,4 & 26,4 & 32,2 & 10,2 & 4,1 & 3,1 & 3,0 \\
\hline $25-50 \mathrm{~cm}$ & 27,5 & 24,6 & 33,0 & 10,7 & 4,2 & 2,4 & \\
\hline $50-80 \mathrm{~cm}$ & 26,1 & 25,3 & 33,9 & 10,5 & 4,2 & 1,8 & \\
\hline
\end{tabular}

qu'elles aient été tassées par des façons de reprise ou d'épandage ( ${ }^{1}$ ).

De façon à obtenir, dans chacun des traitements, un état structural le plus homogène possible, le labour a été réalisé avec une charrue monosoc. Dans la suite du travail, nous supposerons que l'examen sur une largeur de soc de charrue ( $35 \mathrm{~cm}$ environ) d'un profil de la couche de sol labouré, permet de repérer l'essentiel de la variabilité spatiale de l'état structural créé par l'outil et nous définissons ainsi une maille d'observation de l'état physique. Les différences enregistrées entre mailles n'étant pas systématiquement liées à l'action du corps de charrue, sont supposées être aléatoires.

Pour établir la représentation morphologique des deux couches, telle qu'elle est réalisée sur la figure 1, nous avons utilisé la méthode de description proposée par MANICHON (1982a et $b$ ). Celui-ci caractérise des unités morphologiques (ensembles structuraux considérés comme homogènes à l'échelle centimétrique à décimétrique) par deux critères : l'état interne des éléments structuraux et leur mode d'assemblage.

L'état interne peut revêtir deux modalités, symbolisées par les lettres $\Delta$ et $\Gamma$, dont les morphologies respectives sont décrites au tableau 2. Des mesures ont établi que l'état $\Delta$ correspondait à une compacité forte et homogène ; elle est plus faible et plus variable pour l'état $\Gamma$. Le mode d'assemblage revêt les 4 modalités décrites au tableau 2. A même état interne, le classement de porosité structurale est le suivant :

$$
\mathrm{M}<\mathrm{SD}<\mathrm{SF}<\mathrm{F} .
$$

La caractérisation structurale d'une couche travaillée est faite à l'aide d'une représentation cartographique des unités morphologiques observées sur la face verticale d'une fosse suffisamment large (4 longueurs de corps de charrue). Les vides centimétriques y sont cartographiés de façon distincte. Le planimétrage des différentes unités permet de calculer la proportion volumique de chacune d'elles.

La répartition des unités morphologiques dans les deux types de couche labourée (tabl. 3), ainsi que les représentations cartographiques de la figure 1 , traduisent bien les différences décrites plus haut.

\section{B. Modes de compactage employés}

Deux modes ont été utilisés, chacun présentant deux intensités d'action. Le premier a été obtenu par un et

(1) Cet auteur explicite les différentes manières d'aboutir à chacune d'elles. 


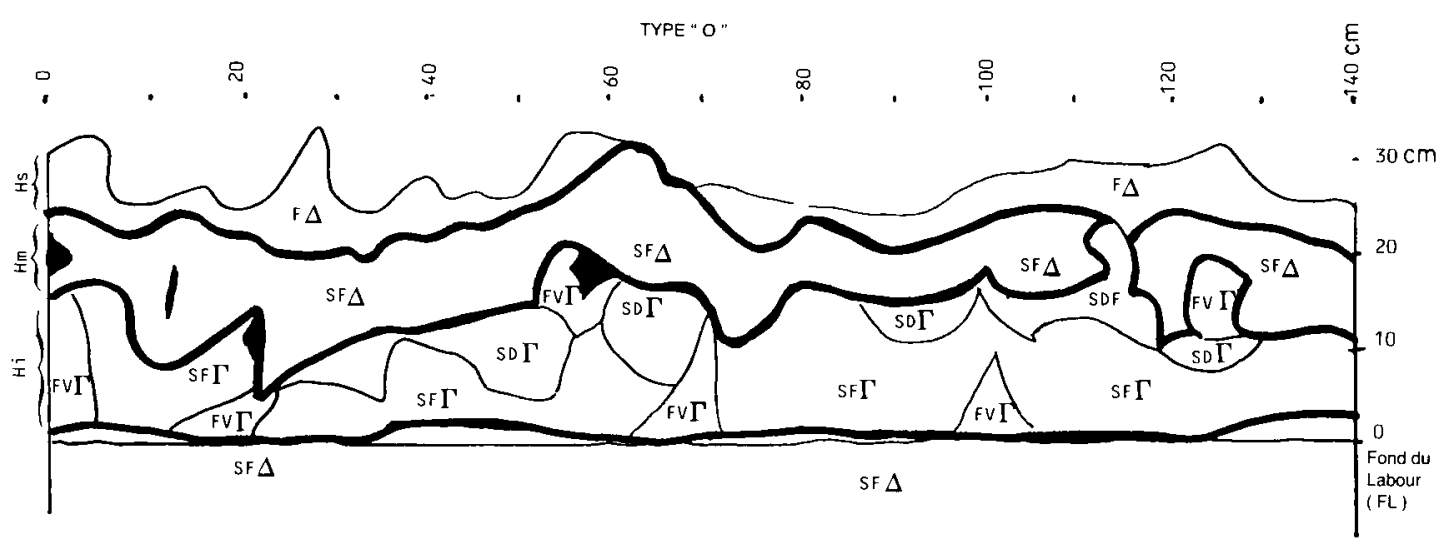

TYPE " B "

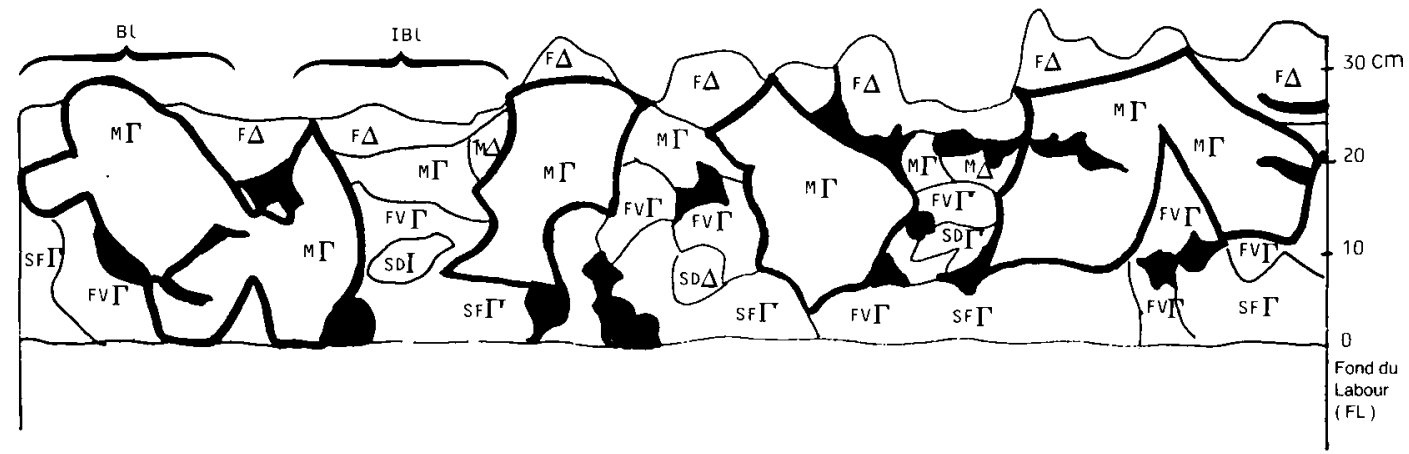

LEGENDE

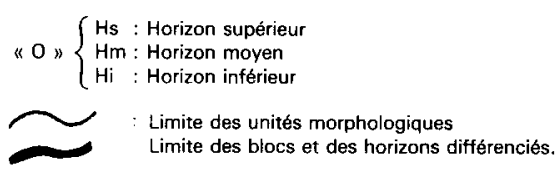

«B* $\left\{\begin{array}{l}B L: \text { Bloc } \\ \mathrm{IBL}: \text { Interbloc }\end{array}\right.$

1 Vide centimétrique.

Figure 1

Cartographie des deux types de couches labourées.

Mapping of two types of ploughed layer.

Note : La signification des symboles caractérisant les unités morphologiques est résumée au tableau 2.

TABLEAU 2

Etats internes des éléments structuraux et modes d'assemblage (d'après MANICHON, 1982a).

Internal states of the structural elements and modes of assemblage.

Identification

Symboles

\begin{tabular}{|c|c|}
\hline $\begin{array}{l}\text { - Face de fragmentation non rugueuse, de forme conchoidale } \\
\text { - Arêtes vives } \\
\text { Forte compacité }\end{array}$ & $\Delta$ \\
\hline $\begin{array}{l}\text { - Face de fragmentation rugueuse } \\
\text { - Arêtes émoussées } \\
\text { - Porosité plus élevée que } \Delta\end{array}$ & $\Gamma$ \\
\hline $\begin{array}{l}\text { - Massif ( } 1 \text { seul élément structural) } \\
\text { - Eléments soudés difficilement discernables } \\
\text { - Eléments soudés facilement discernables } \\
\text { - Eléments individualisés ; assemblages fragmentaires } \\
\text { - Variantes par rapport aux états F, SF, SD, indiquant la présence de vides importants } \\
\text { de l'ordre de plus de } 5 \mathrm{~mm} \text {, mais inférieurs au cm }\end{array}$ & $\begin{array}{l}\text { M } \\
\text { SD } \\
\text { SF } \\
\text { F } \\
\text { FV, SFV, } \\
\text { SDV }\end{array}$ \\
\hline — Les vides centimétriques sont identifiés à part & $\mathrm{v}$ \\
\hline
\end{tabular}


TABLEAU 3

Masse volumique moyenne (pd en g.cm') et répartition des unités morphologiques dans les deux types de couche labourée. Mean bulk density ( $\rho$ d in g ' cm') and distribution of morphological units in the two types of ploughed layers.

\begin{tabular}{|c|c|c|c|c|c|c|c|c|c|c|}
\hline Type de couche & $\rho d$ & $\mathrm{M \Gamma}$ & $\mathrm{SD} \Delta$ & $\mathrm{SD \Gamma}$ & $\mathrm{SF} \Delta$ & $\mathrm{SF} \Gamma$ & $\mathrm{F} \Delta$ & $\mathrm{F} \Gamma$ & $\mathrm{F} \Gamma \mathrm{V}$ & V \\
\hline Couche $\mathrm{O}$ & $<1$ & 0 & 0 & 9,8 & 20,1 & 35,1 & 20,2 & 3,0 & 10,1 & 1,7 \\
\hline \multirow[t]{2}{*}{ Couche B } & 1,0 & \multicolumn{2}{|c|}{$48,4\left(^{1}\right)$} & 5,4 & 0 & 11,4 & 9,2 & 4,6 & 12,1 & 8,9 \\
\hline & & \multicolumn{2}{|c|}{ Blocs } & \multicolumn{7}{|c|}{ Interblocs } \\
\hline
\end{tabular}

(1) Les blocs sont constitués par des ensembles MT et SD $\Delta$.

TABLEAU 4

Caractéristiques du mode de compactage par roulage. Characteristics of compaction by wheels.

\begin{tabular}{|c|c|c|c|}
\hline Tracteur : MF 140, sans outils & $\begin{array}{l}\text { Largeur et pressi } \\
\text { roues }\end{array}$ & $\begin{array}{l}\text { s de gon } \\
\text { Avant }\end{array}$ & $\begin{array}{l}\text { e des } \\
\text { Arrière }\end{array}$ \\
\hline Vitesse : $4 \mathrm{~km} / \mathrm{h}$ & Largeur $(\mathrm{m})$ & 0,20 & 0.35 \\
\hline Nomore de passages: 1 el 3 & $\begin{array}{l}\text { Pression de gon- } \\
\text { flage }(\mathrm{k} \mathrm{Pa})\end{array}$ & 200 & 140 \\
\hline
\end{tabular}

La trace longitudinale des roues arrières étant de $30 \mathrm{~cm}$ environ, pour une vitesse d'avancement de $4 \mathrm{~km} / \mathrm{h}$, la durée d'application de la pression esi de $0,3 \mathrm{~s}$. Pour la roue avant, elle esi plus faible

trois passages de tracteur (tabl. 4), réalisés perpendiculairement au sens des labours. Ce type de traitement exigeant beaucoup de place sur le terrain, nous avons utilisé un second procédé, qui permet de multiplier plus facilement les expériences. Il consiste en une machine à compacter, constituée par une plaque d'appui actionnée par un vérin hydraulique double effet. Ce dernier est monté sur un bâti rigide s'adaptant au système de fixation trois points des tracteurs agricoles (1). La plaque d'appui rectangulaire a une longueur égale à la largeur des bandes de labour. En l'appliquant perpendiculairement au labour, on réalise un compactage sur une maille élémentaire, prise comme échantillon représentatif de l'organisation structurale de la couche labourée. Les caractéristiques de ce dernier mode de compactage sont données au tableau 5. Les pressions appliquées sont voisines de celles du compactage par roulage, mais la durée d'application est beaucoup plus grande.

\section{Mesures réalisées pour caractériser I'état physique}

L'état hydrique est caractérisé par l'humidité moyenne de la couche labourée. Ayant montré que la variation d'humidité pondérale en son sein était largement liée aux hétérogénéités et discontinuités de structure (PAPY, 1986a), nous déterminons la teneur pondérale de la couche sur une maille élémentaire par prélèvements de 4 carottes contiguës sur une largeur de soc de charrue; nous intégrons ainsi dans la valeur

( $\left.{ }^{1}\right)$ Cette machine est semblable à celle mise au point à la station de Science du Sol d'Avignon. INRA - Domaine St Paul, BP 91, 84140 Montfavet (J. GuERIF).
TABLEAU 5

Caractéristiques du mode de compactage par machine (Vérin hydraulique el plaque d'appui).

Characteristics of compaction by machine.

\begin{tabular}{cccc}
\hline Symbole & Force $(\mathrm{N})$ & $\mathrm{S}\left(\mathrm{m}^{2}\right)$ & $\begin{array}{c}\text { Pression } \\
\text { exercee }(\mathrm{k} \mathrm{Pa})\end{array}$ \\
\hline P1 & 12000 & 0,08 & 150 \\
P2 & 18000 & 0,08 & 225 \\
\hline
\end{tabular}

Le temps d'application choisi est de $20 \mathrm{~s}$

moyenne l'ensemble des unités morphologiques dans une proportion supposée être celle de la couche labourée.

L'état structural est caractérisé selon la méthode indiquée ci-dessus, qui permet de quantifier la répartition dans la couche des différentes morphologies présentes. Cette méthode morphométrique a l'inconvénient d'être longue à appliquer. Dans les essais de compactage par roulage, où elle a été utilisée, nous n'avons pu caractériser ainsi qu'une maille correspondant à une largeur de charrue. Etant donné l'hétérogénéité des mailles entre elles, les résultats seront interprétés avec prudence.

Dans les essais de compactage à la machine, l'état structural obtenu a été caractérisé par la masse volumique obtenue par atténuation $\gamma$ (SOANE et al., 1971). Elle a été mesurée sur une largeur correspondant à la maille élémentaire, à 4 niveaux à partir du niveau du sol après compactage $(5,10,15$ et $20 \mathrm{~cm})$. Pour chaque traitement le sondage a été répété 6 fois.

Le volume massique des mottes variant avec leur teneur en eau, pour caractériser l'espace poral en s'affranchissant de cette variation, il faut distinguer porosité structurale et texturale (STENGEL, 1979). Sur de petits agrégats d'état interne $\Delta$, calibrés entre 2 et $3 \mathrm{~cm}$ et prélevés dans le sol étudié, M. FIES (communication personnelle) a établi les droites de retraitgonflement en régime de dessiccation. Elles sont représentées dans la figure 2 , dans un système de coordonnées $\left(\vartheta, \mathrm{e}_{\mathrm{T}}\right)$ où :

$\vartheta=$ indice d'eau, c'est-à-dire le volume d'eau sur le volume solide (1),

(1) $\vartheta=\mathrm{w} . \rho s / \rho \mathrm{w}$, avec : $\mathrm{w}=$ Humidité pondérale ; $\rho \mathrm{s}=$ Masse volumique de la phase solide (ici $2,68 \mathrm{~g} / \mathrm{cm}^{3}$ ); $\rho w=$ Masse volumique de l'eau (supposée égale à $1 \mathrm{~g} / \mathrm{cm}^{3}$ ). 


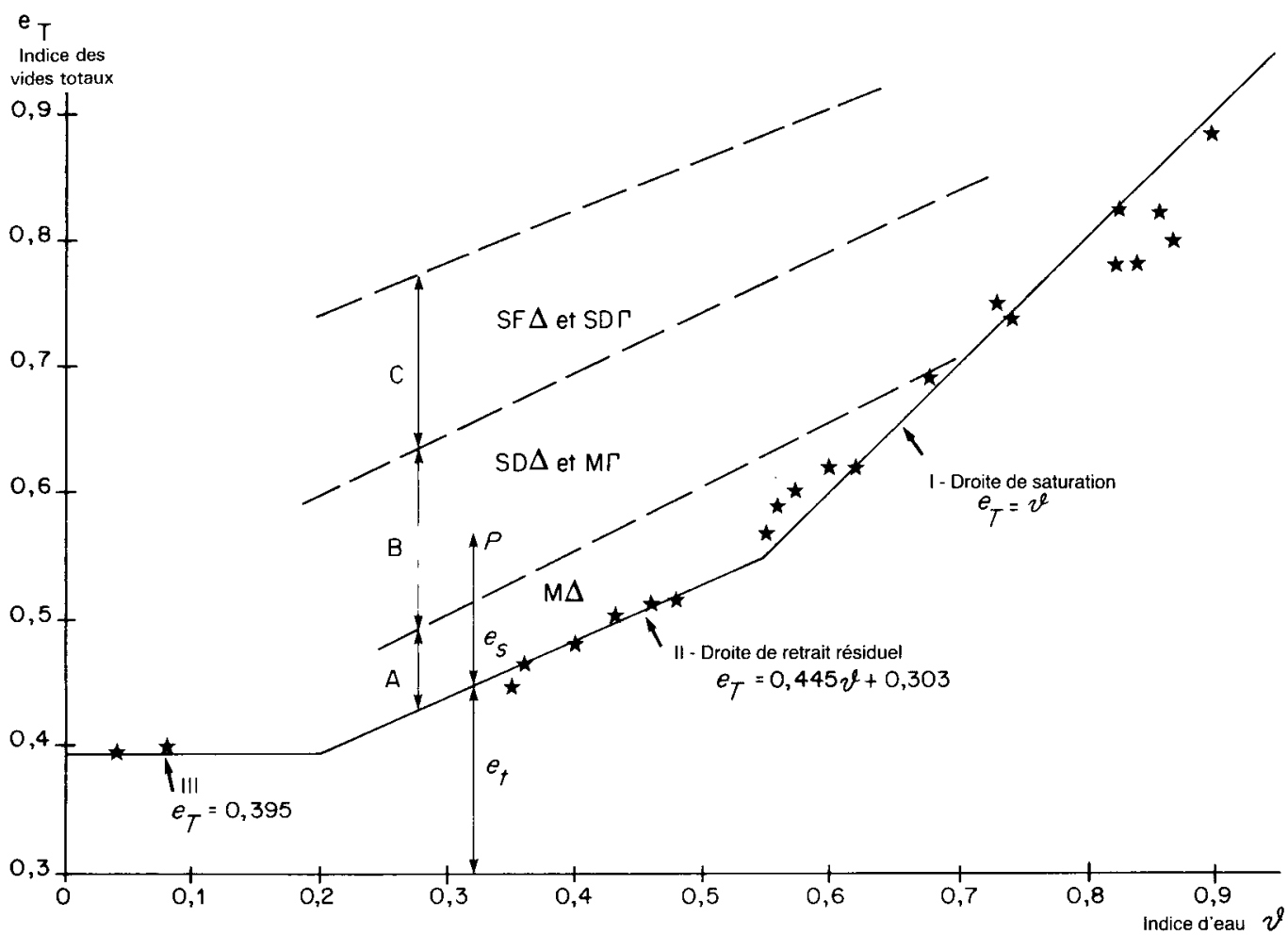

Figure 2

Droites de retrait $(I, I I, I I)$ établies sur de petits agrégats $\Delta$. Zones des points figuratifs des unités morphologiques $M \Delta, M \Delta, S D \Delta, S D \Delta$. Shrinkage curves $(1, I I$, III) established for small aggregates $\Delta$. Zones corresponding to the morphological units $M \Delta . M \Gamma, S D \Delta, S D \Gamma$.

$\mathrm{e}_{\mathrm{T}}=$ indice des vides totaux, c'est-à-dire le volume poral total sur le volume solide.

Au-dessus de $\vartheta=0,54(\mathrm{w}=0,20 \mathrm{~g} / \mathrm{g})$, la droite de retrait-gonflement se confond avec la droite de saturation d'équation :

$$
\mathrm{e}_{\mathrm{T}}=\vartheta \text { (Droite I, fig. 2). }
$$

Pour des valeurs de $\vartheta$ comprises entre 0,19 et 0,54 , il existe un retrait résiduel (Droite II, fig. 2) (1). Audessous d'un indice d'eau de 0,19 , il n'y a pas de variation d'indice des vides (Droite III, fig. 2).

L'ensemble des travaux récents en la matière conduisent à penser que la morphologie $\Delta$ correspondrait à la porosité texturale (MANICHON, 1986). Les trois segments de droite de la figure 2 représenteraient donc la variation d'indice des vides qui la caractérise.

Sur des échantillons de mottes représentatifs des unités morphologiques, nous avons, pour différentes teneurs en eau, déterminé la masse volumique par enrobage à la paraffine ${ }^{(2)}$. On peut ainsi, pour chaque échantillon, déterminer les coordonnées $\vartheta$ et $\mathrm{e}_{\mathrm{T}} \mathrm{du}$ point figuratif $\mathrm{P}$ qui la caractérise (fig. 2). L'ordonnée $\mathrm{e}_{\mathrm{T}}$ de ce point est la somme de l'indice des vides structuraux $\left(e_{s}\right)$ et de l'indice des vides texturaux $\left(e_{t}\right)$ de l'échantillon. L'ensemble des points figuratifs des échantillons prélevés se répartissent dans le système de coordonnées retenu en fonction du type d'unité morphologique, comme indiqué dans la figure 2. Ces résultats établissent qu'on peut obtenir une même valeur de porosité structurale par des combinaisons différentes d'état interne et de mode d'assemblage (zones $\mathrm{B}$ et $\mathrm{C}$ de la fig. 2). La porosité structurale peut donc être décomposée en porosité d'assemblage et porosité structurale d'état interne. Ces résultats, ainsi que ceux obtenus par PELLERIN (1986), valident les observations visuelles pour établir des classements de porosité.

\section{L'Enfoncement}

L'Enfoncement (Enf. en cm) dû au compactage a été mesuré par différence des niveaux moyens établis par rapport à un point de cote fixe. Il est pris comme un indicateur de la déformation subie par la couche labourée. pi représentant la profondeur initiale de cette couche, sa valeur après compactage est pi-Enf, sous hypothèse que la cote du fond de labour n'ait pas été abaissée. A l'aplomb d'une surface de section $\mathrm{S}$ d'application de la pression, la masse de terre contenue dans la couche après compactage est :

$$
\rho d \text { (pi-Enf) . S, }
$$

$\rho d$ étant la masse volumique moyenne. Une diminution de cette masse est l'indice d'un déplacement latéral de terre, phénomène appelé fluage. La mesure de l'enfoncement et de la masse volumique moyenne permet d'en déterminer l'importance.

(1) L'existence d'un retrait résiduel dans les bornes indiquées est tout à fait conforme aux résultats obtenus sur d'autres matériaux de granulométrie identique (FiEs, communication orale).

(2) On trouvera le mode opératoire de cette méthode (mis au point à la station de Science du Sol d'Avignon) dans MANichoN, $1982 a$. 


\section{EFFET DU COMPACTAGE EN FONCTION DE L'ÉTAT HYDRIQUE}

C'est sur la couche 0 que cet effet a été le mieux étudié (plus grand nombre d'états hydriques testés). Nous présentons les résultats s'y rapportant, en commençant par le mode de compactage le moins sévère.

\section{A. Résultats}

\section{Résultats du compactage par roulage}

Le roulage a été fait à des teneurs moyennes en eau de la couche labourée de 0,23 et $0,26 \mathrm{~g} / \mathrm{g}$. Elles correspondent à des durées d'attente, respectivement de 8 et 4 jours, après qu'une pluie a ramené la couche labourée à la capacité au champ. Pour faire une caractérisation morphométrique du résultat du compactage, nous avons réalisé dans les traces de roues de tracteur, perpendiculairement à la direction d'avancement, des observations de profil dans 4 plans verticaux équidistants, répartis sur une distance totale égale à la largeur d'un soc de charrue. La figure 3 est un exemple de bloc de diagramme ainsi décrit.

Pour chacun des 2 niveaux de compactage (1 et 3 passages) et d'états hydriques, on obtient par planimétrie la proportion des différentes unités morphologiques (tabl. 6). Par comparaison aux résultats du tableau 3, on peut caractériser les évolutions de l'état structural sous les différentes actions appliquées.

Ces dernières n'ont pas modifié la nature de l'état interne. Initialement la somme des unités morphologiques à éléments $\Delta$ est de 40,3 p. 100 (d'après le tabl. 3). Après un passage de tracteur, elle prend les valeurs de 43,3 et 36,2 p. 100 pour des teneurs en eau respectives de 0,23 et $0,26 \mathrm{~g} / \mathrm{g}$. Après 3 passages aux mêmes humidités, les unités à éléments $\Delta$ représentent respectivement 35,4 et 40,0 p. 100 .

Le mode d'assemblage, par contre, est fortement affecté (fig. 4).

Les valeurs moyennes de la masse volumique obtenue après les passages de tracteur sont reportées sur la figure $5-I$.

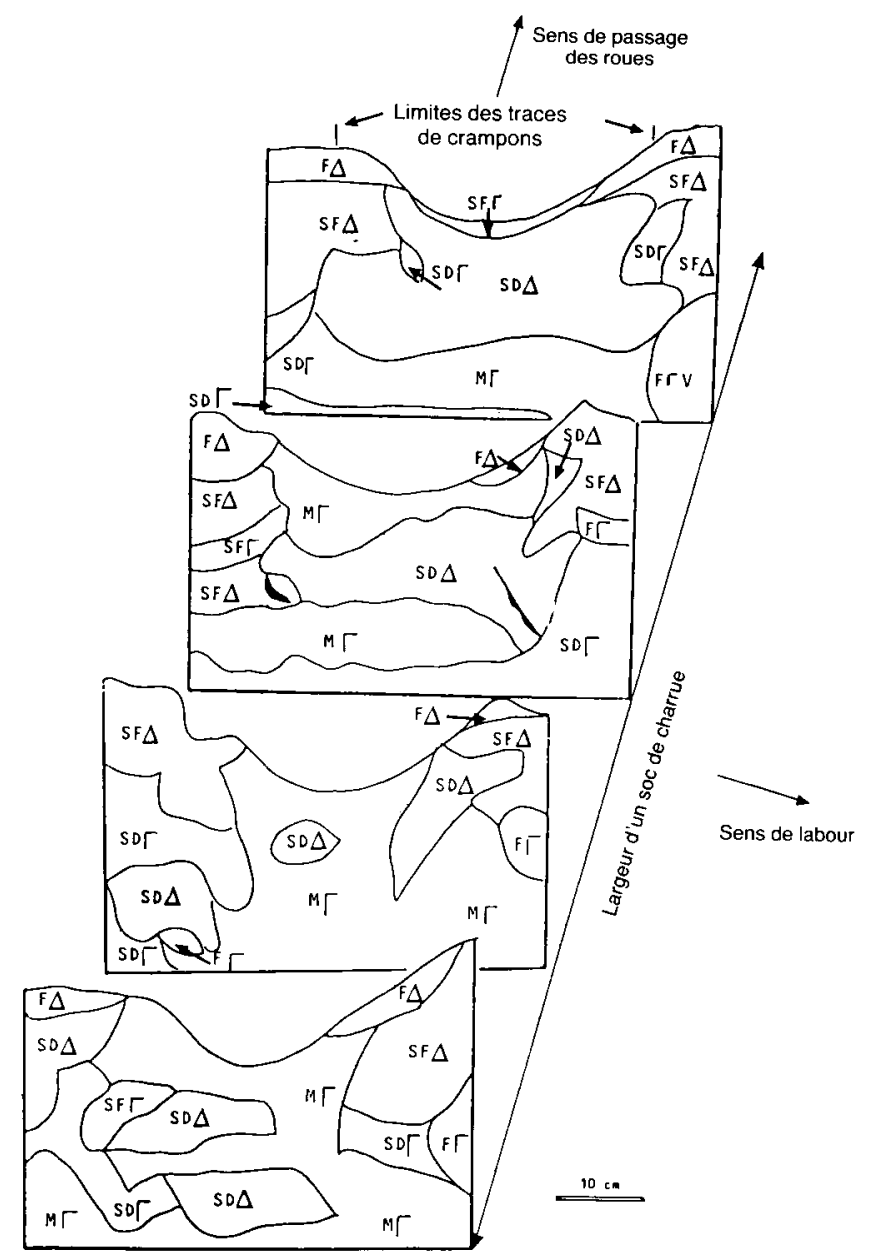

Figure 3

Cartographie d'une maille élémentaire après compactage par roulage de la couche " $O$ " (3 passages de tracteur; $w=26 \mathrm{p} .100)$. $\triangle$ Vide centimétrique

Profile mapping obtained after compaction by wheel in ploughed layer " $O$ " 3 passages: $w=26 \%)$.

Note : La signification des symboles caractérisant les unités morphologiques est donnée au tableau 2.

\section{Résultats du compactage à la machine}

Sur la figure 5-I, sont également indiquées les valeurs de masse volumique obtenues dans l'essai de compactage à la machine. Les intervalles de confiance

TABLEAU 6

Masse volumique moyenne ( $\rho$ d en $\mathrm{g} / \mathrm{cm}$ ) et répartition des unités morphologiques après compactage par roulage sur la couche labourée de type « O », en fonction du nombre de passages $(n)$ et de l'humidité moyenne de la couche ( $w \%$ ).

Mean bulk density $\left(\rho d\right.$ in $\left.\mathrm{g} / \mathrm{cm}^{3}\right)$ and distribution of morphological units after compaction of type " $O$ " ploughed layer according to number of passages $(n)$ and water content $(w \%)$.

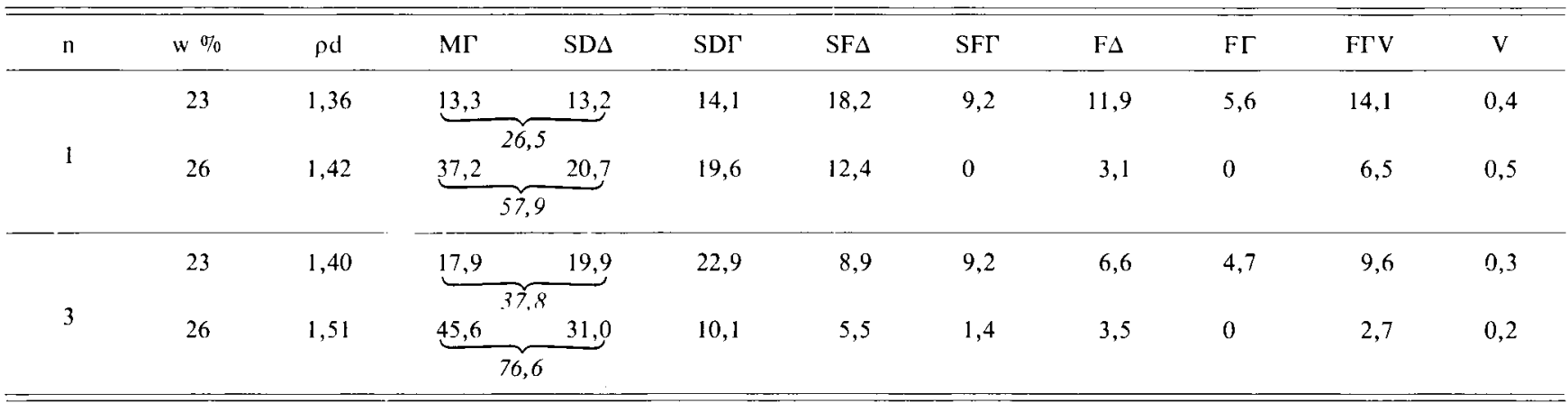




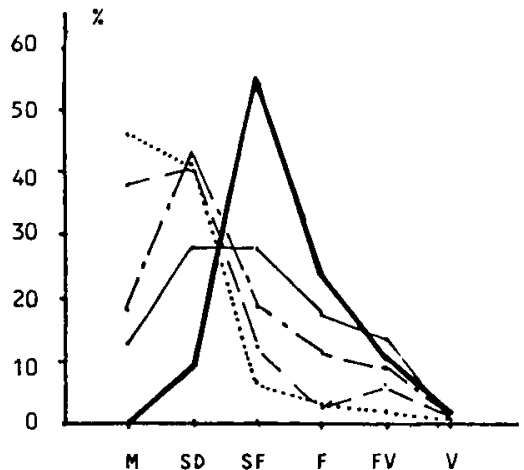

\begin{tabular}{|c|c|c|}
\hline $\begin{array}{l}n \\
w \div\end{array}$ & $\begin{array}{l}\text { cmbre de } \\
\text { umidité }\end{array}$ & sassages \\
\hline$w \%$ & 1 & 3 \\
\hline 26 & --- & ...... \\
\hline 23 & $\longrightarrow$ & $-\cdot-$ \\
\hline
\end{tabular}

Figure 4

Répartition des p. 100 des modes d'assemblage et des vides rentimétriques après compactage, comparée à celle de l'état initial.

Distribution of assemblage modes after compaction compared with initial distribution.

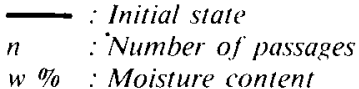

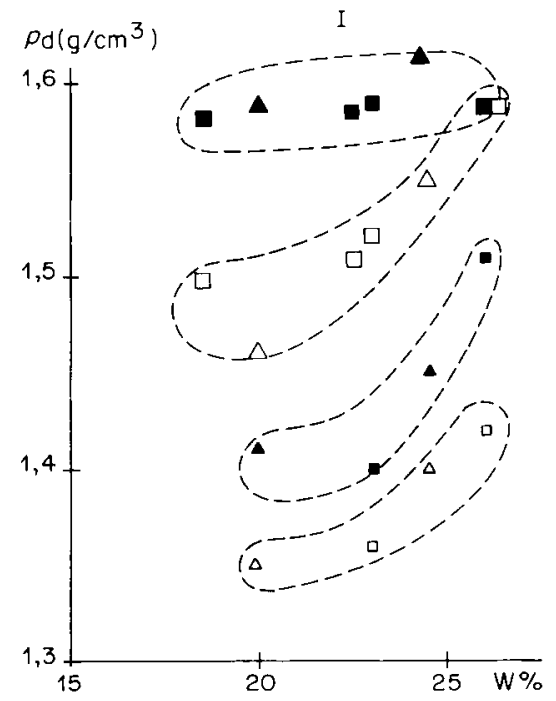



Figure 5

Variation de la masse volumique moyenne (I) et de la masse de terre/ $\mathrm{cm}^{2}$ de surface (II) après compactage en fonction de l'humidité au moment de l'opération ( $w \%$ ).

Variation of mean bulk density (I) and mass of soil $\mathrm{cm}^{2}$ of area (II) according to moisture content before compaction (w \%).

Type de couche $O \quad B$

(Type of ploughed layer)

\begin{tabular}{|c|c|c|c|}
\hline Essai roulage & $\int I$ passage & D & $\Delta$ \\
\hline (Wheel compaction) & 3 passages & - & 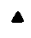 \\
\hline Essai à la machine & $P I$ & $\square$ & $\therefore$ \\
\hline (Machine compaction) & $P 2$ & $\square$ & $\Delta$ \\
\hline
\end{tabular}

$p d$ : Masse volumique moyenne de la couche après compactage (Mean bulk density of ploughed layer after compaction)

pi : Profondeur initiale de la couche labourée (Initial ploughed layer depth)

Enf: Enfoncement

(Shrinkage) 


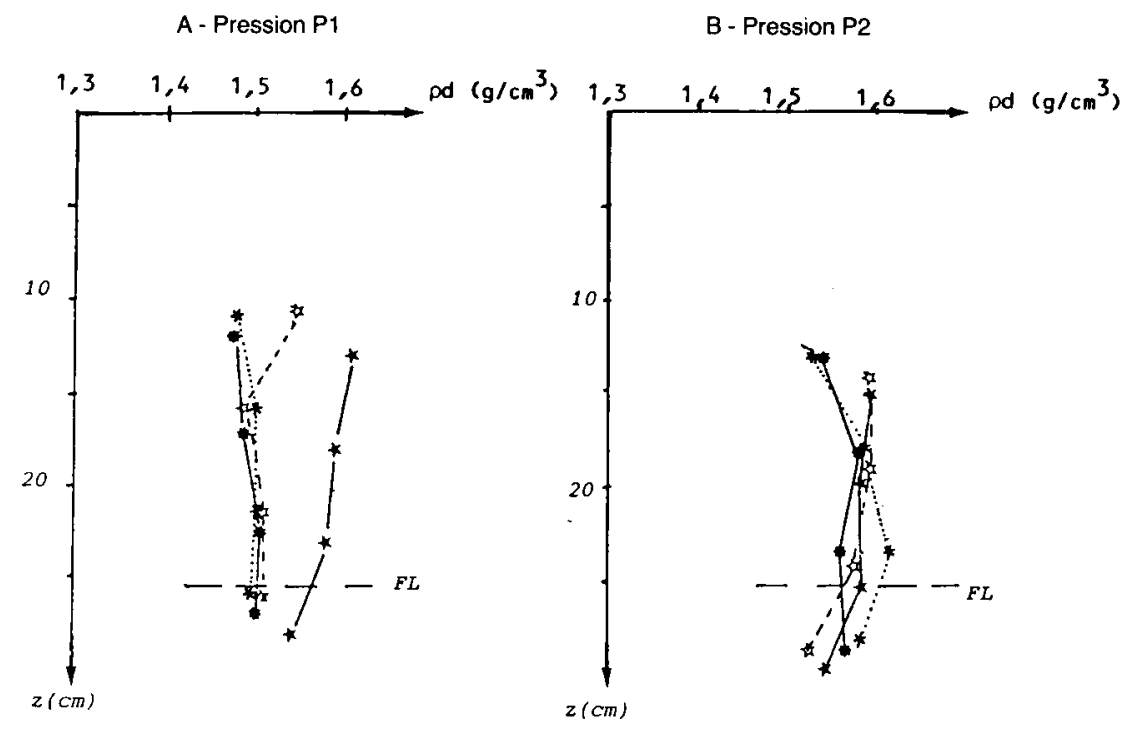

Figure 6

Profil de masse volumique après compactage à différents états hydriques.

Profiles of bulk density after compaction at different water contents.

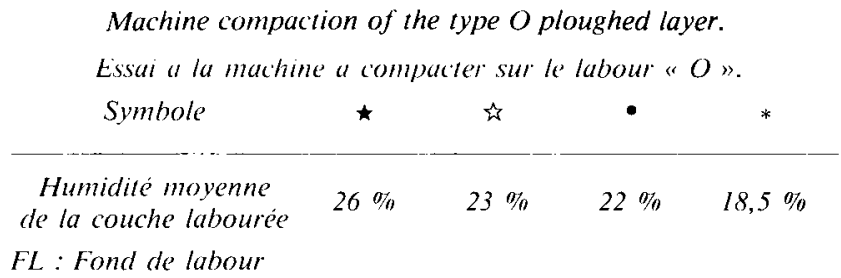

calculés à partir des mesures réalisées sur 6 mailles élémentaires (de $\pm 0,05$ à $\pm 0,1$ ) mettent en évidence une assez forte dispersion. Elle est vraisemblablement due à la variabilité dans l'espace des états structuraux initiaux aux emplacements des placettes compactées.

La figure 5-II donne la variation de la masse de terre contenue dans la couche labourée après compactage par $\mathrm{cm}^{2}$ de surface [od (pi-Enf)] en fonction de l'humidité au moment de l'opération. L'écart à la valeur initiale des valeurs obtenues est un indice de fluage.

Les profils de masse volumique établis à la sonde $\gamma$ sont donnés dans la figure 6 . Ils caractérisent les états finaux obtenus à la verticale de la plaque d'appui. Des observations de profils réalisés après le compactage à la machine ont permis de délimiter l'extension du tassement latéral, manifestation d'un fluage plus ou moins poussé (fig. 7). Tous les compactages à la pression $\mathrm{P} 2$ et celui réalisé à la plus forte humidité à la pression P1 ont abouti, sur l'ensemble de la couche, à un état $\mathrm{M} \Delta$. Aux humidités plus faibles, la pression P1 a donné des modes d'assemblage $M$ dominants et $\mathrm{SD}$ avec une augmentation manifeste, mais non quantifiée d'état interne $\Delta$, par rapport à l'état initial.

\section{B. Discussion}

Une remarque préliminaire s'impose. On sait qu'au fur et à mesure que sèche la couche labourée, le gradient hydrique augmente. Notre dispositif ne permet pas de dissocier l'effet humidité moyenne de cette couche de l'effet gradient dont GUERIF (1983) a mon- tré l'influence : il a mis en évidence un effet protecteur sur le résultat du compactage de la présence dans les premiers centimètres de surface d'une couche sèche.

Les résultats de la figure 5-I montrent :

1) qu'à même humidité, les niveaux de compactage caractérisés par la masse volumique moyenne obtenue, se classent comme suit :

$$
1 \text { passage }<3 \text { passages }<\mathrm{P} 1<\mathrm{P} 2 \text {. }
$$

2) que pour chacun de ces niveaux, la masse volumique moyenne obtenue augmente avec l'humidité. Il existe une valeur limite, atteinte après compactage à la pression P1 pour la plus forte teneur en eau, et à la pression P2 quelle que soit l'humidité dans la gamme testée.

Parallèlement on constate que l'indice de fluage tel qu'il est représenté sur la figure 5-II, nul à faible pour le compactage à la pression $\mathrm{P} 1$, augmente avec l'humidité pour la pression $\mathrm{P} 2$. Ainsi il apparaît que le déplacement latéral de terre ne se manifeste nettement que lorsqu'à la verticale de la surface d'application un certain niveau limite de tassement est atteint.

L'ensemble de nos résultats nous permet de proposer un schéma général du comportement de la couche labourée pour des niveaux croissants de déformations dues à l'humidité du sol et à l'énergie appliquée.

Dans les essais roulage nous avons observé, à partir d'un état initial caractérisé par une absence de mode d'assemblage de type $M$ et peu de type SD (tabl. 3), une augmentation de ces deux modalités avec le nombre de passages de tracteurs et la teneur en eau 

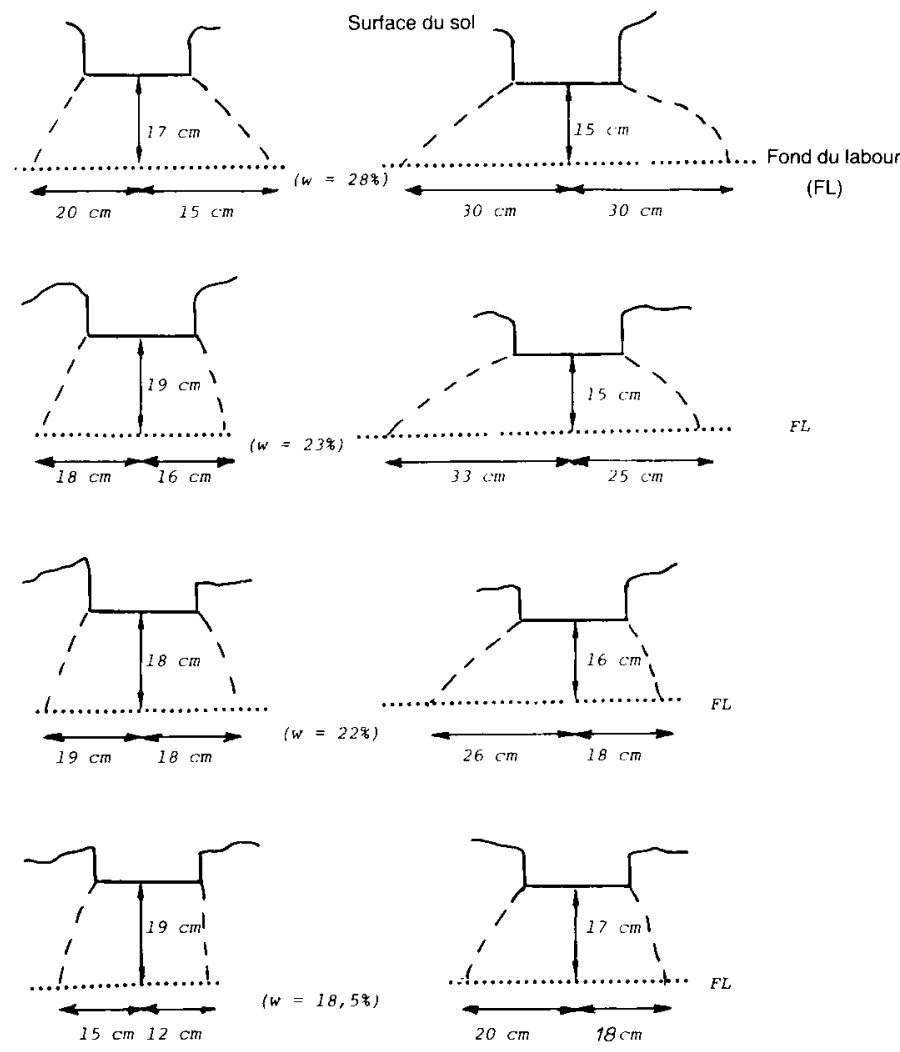

(tabl. 6, fig. 4). Aucune variation systématique d'état interne ni d'indice manifeste de fluage n'ont été observés.

Dans les compactages à la machine, pour les pressions et les humidités les plus faibles, on n'a observé que des modes d'assemblage de type $\mathrm{M}$ et $\mathrm{SD}$; ce dernier disparaît pour le compactage à la pression $\mathrm{P} 1$, à 26 p. 100 de teneur en eau, ainsi que dans toutes les situations correspondant à $\mathbf{P} 2$. Toute la couche compactée est alors massive. L'état interne est également nettement modifié dans ces essais à la machine. Alors que le fond de la couche 0 est initialement constitué de SF $\Gamma$ et SD $\Gamma$ (cf. fig. 1), l'observation n'a mis en évidence que du $M \Delta$ après les compactages les plus sévères ( $\mathrm{P} 2$ et $\mathrm{P} 1$ aux fortes teneurs en eau). La figure 6 montre que les valeurs de masses volumiques correspondant à cette morphologie voisinent $1,6 \mathrm{~g} / \mathrm{cm}^{3}$.

On peut synthétiser comme suit l'évolution des manifestations du compactage au fur et à mesure qu'augmente la déformation du système en fonction de l'humidité et de l'énergie appliquée. Il y a d'abord rapprochement des éléments structuraux jusqu'à obtention d'un état massif ; à partir de cet état limite, on enregistre une modification de l'état interne (transformation d'éléments $\Gamma$ en $\Delta$ ) en même temps que la terre flue à l'aplomb des surfaces d'application des charges. Une fois l'état $\mathbf{M} \Delta$ obtenu, toute augmentation de compactage se traduit par un fluage d'autant plus marqué que l'humidité et l'énergie appliquée sont plus fortes. On met ainsi en évidence des états seuils au-delà desquels la nature de la déformation se modifie.

Notons qu'on peut obtenir de plusieurs manières un même état final. Les mesures et les observations de
Figure 7

Variation du volume tassé observé sur un profil de sol en fonction de la pression et de l'état hydrique au moment du compactage.

Note : Les cotes figurées sont des valeurs moyennes établies à partir de plusieurs observations de profils.

$w=$ Humidité moyenne de la couche labourée au moment du compactage

$F L=$ Fond du labour

Variation of packed zones in profiles according to the applied pressure and the water content before compaction.

profils montrent que c'est le cas, par exemple, pour les deux modalités de compactage suivantes: P1 à 26 p. 100 et $P 2$ à 18,5 p. 100.

\section{EFFET DE COMPACTAGE EN FONCTION DE L'ÉTAT STRUCTURAL}

Nous avons montré précédemment que soumis à des séquences identiques de pluie et de dessiccation, en sortie d'hiver, les 2 types de couche $\mathrm{O}$ et $\mathrm{B}$ avaient un comportement hydrique différent (PAPY, 1986a). En B l'infiltration se fait de façon privilégiée par les lacunes entre les blocs; ces derniers s'imbibent moins que des unités morphologiques plus poreuses. Il résulte de ce phénomène que, au printemps, les couches labourées de type $B$ ont des teneurs en eau plus faibles que celles de type $O$. Mais la question se pose de savoir s'il existe un effet propre de l'état structural. C'est ce que nous allons examiner en comparant à ceux obtenus sur $O$ les résultats réalisés dans la couche $B$.

Remarquons que l'étude expérimentale présentée ici ne permet pas une analyse de l'effet de l'état structural aux différents niveaux où on peut le caractériser : état interne, mode d'assemblage, disposition spatiale des unités morphologiques les unes par rapport aux autres. Ils sont ici tous confondus.

\section{A. Résultats}

Les compactages ayant été réalisés à même date sur $\mathrm{O}$ et $\mathrm{B}$, les teneurs en eau correspondantes étaient plus faibles dans le second cas. En reportant sur la 
TABLEAU 7

Masse volumique moyenne ( $\rho d$ en $\left.\mathrm{g} / \mathrm{cm}^{3}\right)$ et répartition des unités morphologiques après compactage par roulage sur la couche labourée de type « $B$ ", en fonction du nombre de passages (n) et de l'humidite moyenne de (a couche (w \%).

Mean bulk density ( $\rho d$ in $\left.\mathrm{g} / \mathrm{cm}^{3}\right)$ and distribution of morphological units after compaction of tvpe " $B$ " ploughed layer according to number of passages $(n)$ and water content $(w \%)$.

\begin{tabular}{|c|c|c|c|c|c|c|c|c|c|c|}
\hline \multirow{2}{*}{$n$} & \multirow{2}{*}{ w $\%$} & \multirow{2}{*}{$\rho d$} & \multirow{2}{*}{$\begin{array}{c}\text { Blocs } \\
(\mathrm{M} \Gamma+\mathrm{SD} \Delta) \\
\left({ }^{1}\right)\end{array}$} & \multicolumn{7}{|c|}{ Interblocs } \\
\hline & & & & SDI & $\mathrm{SF} \Delta$ & $\mathrm{SF} \Gamma$ & $\mathrm{F} \Delta$ & $\mathrm{F} \Gamma$ & FIV & V \\
\hline \multirow{2}{*}{1} & 21,4 & 1,35 & 47,1 & 4,8 & 14,4 & 8,0 & 3,6 & 2,8 & 15,7 & 3,3 \\
\hline & 24,4 & 1,40 & 43,9 & 25,0 & 15,0 & 3,0 & 0,2 & 0 & 8,0 & 4,9 \\
\hline \multirow{2}{*}{3} & 21,4 & 1,42 & 50,7 & 17,9 & 8,7 & 5,6 & 3,4 & 3,5 & 9,6 & 0,6 \\
\hline & 24,4 & 1,45 & 74,4 & 10,9 & 2,8 & 3,1 & 0,8 & 0,4 & 6,5 & 1,1 \\
\hline
\end{tabular}

(1) Au sein des blocs il est difficile de distinguer la limite entre $\mathrm{M} \Gamma$ et SD $\Delta$

figure 5-I les valeurs des masses volumiques moyennes de la couche $B$ après compactage en fonction de l'humidité, on constate les mêmes relations entre variables que dans le cas de la couche $O$.

La description morphologique réalisée après roulage de la couche $B$ par le tracteur permet de calculer la répartition des différentes unités morphologiques (tabl. 7). On distingue dans la couche tassée des blocs continus constitués de $\mathrm{M} \Gamma$ et $\mathrm{SD} \Delta$. Il n'a pas été fait sur B d'observations morphologiques, même sommaires, dans l'essai de compactage à la machine.

\section{B. Discussion}

Ces résultats établis sur $\mathbf{B}$ attestent une similarité avec ceux décrits sur $\mathrm{O}$ : augmentation de la masse volumique et diminution de la porosité d'assemblage avec la teneur en eau et le niveau d'énergie appliquée. Aucune observation morphologique n'ayant été réalisée après compactage à $\mathrm{P} 1$ et $\mathrm{P} 2$, la comparaison ne peut être faite sur l'ensemble de la gamme des énergies appliquées à $\mathrm{O}$.

Des différences de comportements sont cependant à noter entre $\mathrm{O}$ et $\mathrm{B}$. Entre les états structuraux initiaux (tabl. 3) et les états après roulage (tabl. 6 et 7 ), on enregistre une modification de la répartition des unités morphologiques plus forte pour $\mathrm{O}$ que pour $\mathrm{B}$. Dans le premier cas, pour la plus faible humidité au compactage, après un seul passage, la somme des unités $\mathrm{M \Gamma}$ et $\mathrm{SD} \Delta$ passe de 0 à $26,5 \mathrm{p}$. 100 ; dans le second elle n'est pas sensiblement modifiée et reste voisine de 45 p. 100. Une couche de type $O$ se dégrade donc par compactage plus facilement qu'une de type B.

Dans la couche $\mathbf{B}$, le compactage de faible intensité (1 passage) ne réduit pas à zéro les vides centimétriques ; ils occupent encore 3,6 à 4,9 p. 100 . Tout se passe comme si, dans ce type structural de couche labourée où blocs et interblocs sont subverticaux, les zones de plus forte cohésion (les blocs) jouaient un rôle de pilotis protégeant les interblocs d'un écrasement poussé. Notons que, dans un autre essai non rapporté ici, où les blocs d'une couche de type $B$ étaient inclinés, les vides centimétriques avaient disparu sous l'effet d'un roulage d'intensité voisine. La disposition spatiale d'unités de cohésion différentes semble donc être un élément important du comportement au compactage.
Signalons enfin l'existence d'une interaction : humidité $\times$ énergie de compactage $\times$ état structural initial. Elle apparaît dans la comparaison des tableaux 6 et 7. Aux faibles intensités d'énergie appliquée (1 passage), l'héritage de l'état structural initial est encore visible puisqu'on distingue bien dans la couche $\mathbf{B}$ les blocs, interblocs et vides centimétriques. Mais à énergie et humidité élevées ( 3 passages à l'état le plus humide), les résultats obtenus à partir de chacun des types de couche convergent vers un même état structural caractérisé par une forte proportion d'unités $\mathrm{M} \Gamma$ et $\mathrm{SD} \Delta$ (plus de 70 p. 100).

Ces résultats ont des conséquences pratiques importantes. TARDIEU et MANICHON (1986) ont montré, en effet, que la présence, dans un horizon travaillé, d'unités morphologiques compactes peut avoir un effet défavorable sur la répartition de l'enracinement dans l'ensemble du profil et ce, d'autant plus que leur volume est grand et leur contact avec le fond du labour étroit. Le rendement des cultures s'en trouve affecté en conditions d'alimentation hydrique déficientes.

Une couche $B$ est plus défavorable à l'enracinement qu'une autre de type $O$, mais le volume des unités morphologiques massives n'étant que de l'ordre du décimètre, et la continuité entre ces zones et le fond du labour n'étant pas généralement assuré, leur effet sur une mauvaise répartition de l'enracinement dans les couches sous-jacentes à l'horizon labouré est relativement faible.

Sous l'effet d'un compactage modéré, l'état de la couche n'est pas sensiblement modifié, tandis qu'un même niveau de compactage affecte beaucoup plus l'état structural d'une couche $O$. Les zones massives créées par le passage des roues de tracteur ont généralement des volumes de plusieurs décimètres et présentent souvent une bonne continuité avec le fond du labour. Les répercussions sur la répartition de l'enracinement sont au niveau des passages de roues plus graves que dans le cas précédent.

\section{CONCLUSION}

Cette étude des effets du compactage d'une couche travaillée a mis en évidence des phénomènes conformes à ceux observés sur un matériel de structure plus homogène : 
- réalisation sous des intensités croissantes de compactage des phénomènes successifs suivants : d'abord resserrement de l'assemblage des éléments structuraux jusqu'à un niveau limite où intervient alors la diminution de leur porosité interne (GUERIF, 1982) ;

- interaction humidité $\times$ énergie appliquée sur la déformation subie par le sol (HUTTER, 1966 ; FAURE, 1978-1980) ;

- interaction humidité $\times$ énergie appliquée $\times$ état structural initial, ainsi que l'ont montré BERTUZZı (1980) par oedométrie, et GRIMALDI (1981) à partir des tests PROCTOR.

Mais dans les conditions de laboratoire très particulières dans lesquelles ces études ont été menées (compactage d'échantillons frettés), la manifestation ultime qu'est le fluage ne pouvait se réaliser.

Les résultats obtenus nous ont permis de proposer un schéma général du comportement de la couche labourée soumise à des niveaux croissants de compactage et de montrer un effet propre de l'état initial de la structure sur la transformation qu'elle subit par compactage.

Ce dernier effet, s'ajoutant à celui que cet état initial a sur le comportement hydrique du sol, se traduit par la nécessité de prendre en compte l'état physique du sol quand on veut préciser les conditions d'intervention culturales, et déterminer les jours disponibles pour le travail du sol (PAPY, 1986b).
Par ailleurs, la pertinence des études morphométriques est apparue dans ce travail. Les observations visuelles, accompagnées de mesures qui en garantissent la fidélité et l'objectivité et permettent d'utiliser comme référence la courbe de retrait établie sur de petits agrégats $\Delta$, constituent un moyen de bien décrire la porosité structurale. Cette dernière, qui est celle qu'affecte le compactage, se compose de porosités d'assemblage et d'état interne. Selon l'intensité d'action c'est l'une ou l'autre qui est affectée. Enfin, un autre intérêt de la quantification morphologique par observation de profils, réside dans la possibilité qu'elle offre de caractériser les manifestations du compactage dans l'espace.

Recu le 20 mai 1986. Accepté le 22 octobre 1986.

\section{REMERCIEMENTS}

Je remercie MM. Manichon et Sebillotte (Chaire d'Agronomie de l'INA-PG), Fies, Guerif, Monnier, Stengel (Laboratoire de Sciences du Sol d'Avignon), pour l'aide qu'ils m'ont apportée dans ce travail.

\section{RÉFÉRENCES BIBLIOGRAPHIQUES}

Bertuzzi P., 1980. Déformation d'un sol argileux par le roulage d'un tracteur : études expérimentales au laboratoire ef in situ. Mémoire de fin d'étude ENITA, sous la direction de P. STENGEL el J. Guerif, $40 \mathrm{p}$.

Faure A., 1978. Comportement des sols au compactage: rôle de l'argile et conséquences sur l'arrangement des grains. Thèse, Grenoble, $179 \mathrm{p}$.

Faure A., 1980. A new conception of the plastic and liquid limits of clay. Soil and Tillage Res, 1, 97-105.

Grimaldi M., 1981. Contribution à l'étude du tassement des sols : évolution de la structure d'un matériau limoneux soumis à des contraintes mécaniques et hydriques. Thèse, Rennes, $221 \mathrm{p}$.

Guérif J., 1982. Compactage d'un massif d'agrégats : effet de la teneur en eau et de la pression appliquée. Agronomie, 2 (3), $287-294$

Guérif J., 1983. The influence of water-content gradient and structure anisotropy on soil compressibility. J. Agric. Enging. Res., 29, 367-374.

Hutter W., 1966. Action des compressions sur la structure du sol. Ann. Agron., 17, 37-52.

Manichon H., 1982a. Influence des systèmes de culture sur le profil cultural: élaboration d'une méthode de diagnostic basée sur l'observation morphologique. Thèse Doct. Ing. INA-PG, 213 p. + Annexes.

Manichon H., 1982b. L'action des outils sur le sol : appréciation de leurs effets par la méthode du profil cultural. Sci. Sol, 3, 203-219.

Manichon H., 1986. Observation morphołogique de l'état structural et mise en évidence d'effets de compactage des horizons travaillés. Séminaire «Land Use », Avignon, 17-18/09/85 (à paraître).
Manichon H., Sebillotte M., 1975. Analyse et prévision des conséquences des passages successifs d'outils sur le profil cultural. Bull. Tech. Inf., 302-303, 569-577.

Papy F., 1984. Comportement du sol sous l'action des façons de reprise d'un labour au printemps. Thèse Doct. Ing. INA-PG, $232 \mathrm{p}$ + Annexes.

Papy F., 1986a. Effet de l'état structural d'une couche labourée sur sa rétention en eau. Agronomie, 6 (6), 555-565.

Papy F., 1986b. La conduite des états physiques du sol cultivé (analyse des effets de l'état initial). Bull. Tech. Inf., 412-413, 685691.

Pellerin S., 1986. Effet de la date de labour sur l'état structural de l'horizon labouré dans les sols argileux gonflants du Marais Poitevin. Agronomie, 6 (7), 683-692.

Soane B. D., Campbell D. J., Herkes S. M., 1971. Hand held gamma ray transmission equipment for the measurement of bulk density of field soil. J. Agric. Enging. Res., 16, 145-156.

Soane B. D., Blackwell P. S., Dickson J. W., Painter D. J., 1981 Compaction by agricultural vehicles : a review.

I - Soil and wheel characteristics. Soil and Tillage Res., 1, 207-237.

II - Compaction under types and other running car. Soil and Tillage Res., 1, 373-400.

Stengel P., 1979. Utilisation de l'analyse des systèmes de porosité pour la caractérisation de l'état physique du sol in situ. Ann. Agron., 30 (1), 27-51.

Tardieu F., Manichon H., 1986. Caractérisation du système racinaire du maïs en tant que capteur d'eau. II - Une méthode d'étude de la répartition verticale et horizontale des racines. Agronomie, $6(5), 415-425$ 